\title{
Multiple-Symbol Differential Sphere Decoding Aided Amplify-and-Forward Differential Space-Time Modulation
}

\author{
Chao Xu, Cong Liu, Soon Xin Ng and Lajos Hanzo \\ School of ECS, University of Southampton, SO17 1BJ, United Kingdom. \\ Email: $\{$ cx1g08,sxn,lh\}@ecs.soton.ac.uk, http://www-mobile.ecs.soton.ac.uk
}

\begin{abstract}
Differential Space-Time Modulation (DSTM) employing multiple antennas provides a diversity advantage in rich scattering environments, whilst the excessive complexity of MIMO channel estimation is avoided. However, due to size, cost or other hardware limitations, the multiple antennas may be in deep fade simultaneously and hence experience spatially correlated fading, which leads to a loss of diversity gain. Hence in this paper, we design an Amplify-and-Forward (AF) relaying aided DSTM scheme, which is capable of attaining a diversity gain even when the source node is in deep fade. Furthermore, MultipleSymbol Differential Sphere Decoding (MSDSD) is developed for the proposed scheme to avoid the potential performance degradation of the noncoherent receiver in fast fading channels.
\end{abstract}

\section{INTRODUCTION}

Multiple-Input Multiple-Output (MIMO) systems, whose transmitters and receivers are equipped with multiple antennas, provide a potentially improved transmission reliability [1] and/or higher capacity [2]. The class of Space-Time Block Codes (STBCs) was proposed for achieving diversity gain [3], [4], where the multiple antennas were employed to transmit redundant information, so that the receiver would be able to have more than a single signal replica, which are unlikely to be faded at the same time. The price paid is that the achievable transmission rate remains the same as that of a single antenna. Hence Linear Dispersion Codes (LDCs) were proposed in [5] in order to strike a flexible trade-off between the achievable throughput and diversity gain. In the LDCs, $Q$ transmitted symbols are dispersed to $M$-spatial and $T$-temporal dimensions, and as a result they exhibit a high design flexibility, which is a benefit of appropriately configuring $Q, M$ and $T$. Due to the fact that the signal processing of MIMO techniques typically involves both the spatial and temporal dimensions, the modulation schemes designed for MIMO systems may be summarized as Space-Time Modulation (STM), which includes both STBCs and LDCs.

However, due to the hardware limitations of shirt-pocket-sized handheld devices, there is often insufficient spacing between the antennas, which leads to spatially correlated fading and as a result, the diversity gain is eroded. To circumvent this problem, cooperative diversity was proposed in [6], [7], where multiple single-element relays may share their antennas to form a Virtual Antenna Array (VAA). Hence it is beneficial for the Base Station (BS) to activate relays, which are sufficiently far apart, so that they may experience independent fading.

Classic coherent receivers require accurate Channel State Information (CSI), but in practice it becomes unrealistic for the relays and the destination to estimate the Channel Impulse Response (CIR) of all the VAA links, especially when the fading channels fluctuate rapidly. As a result, CSI estimation errors may erode the $3 \mathrm{~dB}$ performance

The financial support of the RC-UK under the auspices of the India-UK Advanced Technology Centre as well as of the China-UK $4^{\text {th }}$ generation wireless systems project and that of the European Union under the auspices of the Optimix project is gratefully acknowledged. advantage of coherent MIMO schemes over their noncoherent counterparts. Hence Differential STM (DSTM) schemes including Differential STBCs (DSTBCs) [8], [9] and Differential LDCs (DLDCs) [10], which eliminates the potentially high Doppler-dependent pilot overhead and high-complexity MIMO channel estimation may be preferred in distributed MIMO systems. This is particular so in the context of the relay.

However, the classic Conventional Differential Detection (CDD) based noncoherent schemes generally suffer a $3 \mathrm{~dB}$ performance penalty in the absence of CSI estimation, even when the fading channels do not fluctuate rapidly. Upon increasing the Doppler frequency, a pronounced irreducible error floor is formed. To mitigate this problem, Multiple-Symbol Differential Detecting (MSDD) was proposed for Differential PSK (DPSK) in [11], [12], where a longer detection window $N_{w}>2$ is employed in order to make a joint decision based on multiple received symbols/blocks. However, the detection complexity increases exponentially as $N_{w}$ increases. Hence, as a remedy, Multiple-Symbol Differential Sphere Detection (MSDSD) was introduced for DPSK and DSTM in [13], [14] in order to limit the escalating complexity of MSDD by the employment of sphere decoding. As a further advance, a MSDSD aided cooperative Amplify-and-Forward (AF) scheme using DPSK signalling was proposed in [15].

Against this background, the novel contributions of this paper are:

1) we propose an AF relaying scheme employing DSTM, which attains its diversity gain even when the multiple antennas employed at the source node are spatially correlated and even if they are in a deep fade at the same time.

2) we develop the MSDSD for the proposed cooperative system in order to mitigate the potential performance degradation of the noncoherent receiver, especially in rapidly fading channels.

The proposed system model is portrayed in Section II, while our simulation results are given in Section III, and our conclusions are offered in Section IV.

\section{System OVERVIEW}

The schematic of the proposed system is shown in Fig. 1. For the co-located DSTM scheme operating without relaying, the transmitter and the receiver are equipped with $M$ and $N$ antennas, respectively. For the proposed AF relaying aided DSTM, $R$ single-element relays are activated, and every set of $M$ relays forms a VAA in order to forward the DSTM symbols of the source node.

More specifically, the source node operating in coalition with the $R$ relays form $U=1+R / M$ Antenna Arrays (AAs). In the first DSTM transmission block period, which is referred to as the broadcast interval, the source node transmits DSTM symbols to both the relays and to the destination. In the following $(U-1)$ transmission block periods, which constitute the cooperation interval, the $(U-1)$ VAAs amplify the power of the signal received from the source node and then forward them in the classic AF mode to the destination. 


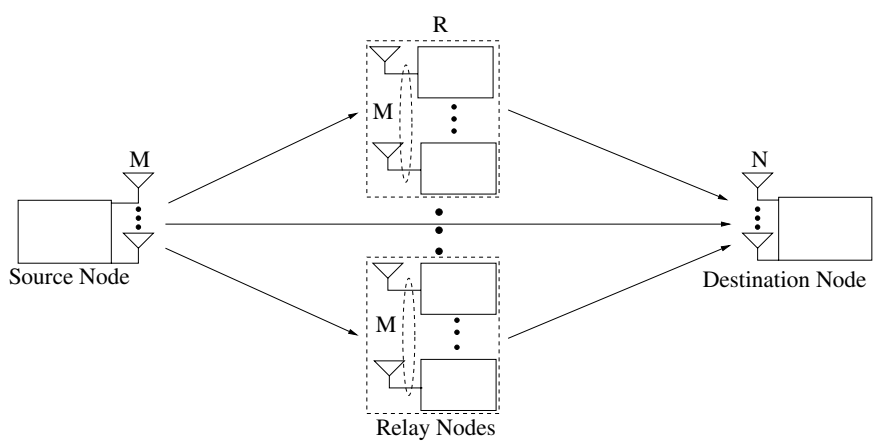

Fig. 1. Block diagram of the proposed amplify-and-forward differetial space time modulation.

We assume that there is no communications among the relays. We also note that a single relay equipped with $M$ antenna elements is capable of replacing a VAA. However, it is unusual to have relays equipped with $M$ antennas, especially when the value of $M$ is high.

In this section, we first portray our system model conceived for the AF relaying aided DSTM scheme, and then develop the MSDSD for the proposed scheme.

\section{A. The AF Relaying Aided DSTM}

1) Broadcast Phase: At the source node, differential encoding is invoked for the DSTM scheme, which is conducted in a way similar to classic DPSK, expressed as [16]:

$$
\mathbf{S}_{n}= \begin{cases}\mathbf{S}_{1} & n=1 \\ \mathbf{X}_{n-1} \mathbf{S}_{n-1} & n>1\end{cases}
$$

where the $(T \times T)$-element unitary matrix $\mathbf{X}_{n}$ maps the information bits to the $(T \times M)$-element DSTM transmit matrix $\mathbf{S}_{n}$. The transmit power at the source node is set to $P_{S}$, while all the $M$ relays of the $u$-th VAA have a transmit power of $P_{R_{u}}$. The total power shared by the source and the relays should be normalized, i.e. we have $P_{S}+$ $\sum_{u=2}^{U} P_{R_{u}}=1$.

Then the signal received at the $m$-th relay of the $u$-th VAA may be formulated as:

$$
\mathbf{Y}_{n}^{S R_{u}^{m}}=\sqrt{P_{S}} \mathbf{S}_{n} \mathbf{H}_{n}^{S R_{u}^{m}}+\mathbf{V}_{n}^{S R_{u}^{m}}
$$

where we have $1 \leq m \leq M$ and $2 \leq u \leq U$, since the first of the $U$ AAs is the source node. The $(T \times 1)$-element matrix $\mathbf{Y}_{n}^{S R_{u}^{m}}$ in Eq. (2) models the signal received from the source at the singleelement relay. The fading channel matrix $\mathbf{H}_{n}^{S R_{u}^{m}}$ of Eq. (2) has $(M \times$ 1) elements, which obey the Rayleigh distribution, while the Additive White Gaussian Noise (AWGN) matrix $\mathbf{V}_{n}^{S R_{u}^{m}}$ has $(T \times 1)$ elements with a zero mean and a variance of $N_{0}^{R_{u}}$ in each dimension.

For the $u$-th VAA, the signal received at all the $M$ relays may be formulated as:

$$
\mathbf{Y}_{n}^{S R_{u}}=\sqrt{P_{S}} \mathbf{S}_{n} \mathbf{H}_{n}^{S R_{u}}+\mathbf{V}_{n}^{S R_{u}},
$$

where $\mathbf{Y}_{n}^{S R_{u}}=\left[\mathbf{Y}_{n}^{S R_{u}^{1}}, \cdots, \mathbf{Y}_{n}^{S R_{u}^{M}}\right]$ has $(T \times M)$ elements. Furthermore, the equivalent fading channel matrix of $\mathbf{H}_{n}^{S R_{u}}=$ $\left[\mathbf{H}_{n}^{S R_{u}^{1}}, \cdots, \mathbf{H}_{n}^{S R_{u}^{M}}\right]$ characterizes the links between the source node and the $u$-th VAA, which has $(M \times M)$ elements. The power of the $u$-th Source-Relay (SR) link is represented by $\sigma_{S R_{u}}^{2}$. The equivalent AWGN matrix at the $M$ relays $\mathbf{V}_{n}^{S R_{u}}=\left[\mathbf{V}_{n}^{S R_{u}^{1}}, \cdots, \mathbf{V}_{n}^{S R_{u}^{M}}\right]$ is of size $(T \times M)$.
Similarly, the destination node also receives signal from the source during the broadcast interval, which is given by:

$$
\mathbf{Y}_{n}^{S D}=\sqrt{P_{S}} \mathbf{S}_{n} \mathbf{H}_{n}^{S D}+\mathbf{V}_{n}^{S D}
$$

where the $(T \times N)$-element matrix $\mathbf{Y}_{n}^{S D}$ models the signal received from the source. The Rayleigh fading channel matrix $\mathbf{H}_{n}^{S D}$ in Eq. (4) has $(M \times N)$ elements and the power of the Source-Destination (SD) link is $\sigma_{S D}^{2}$. The AWGN matrix $\mathbf{V}_{n}^{S D}$ has $(T \times N)$ elements with a zero mean and a variance of $N_{0}^{D}$ in each dimension.

2) Cooperation Phase: During the cooperation interval, the relays normalize the power of the received signal, and then forward it to the destination. The amplification factor at the $u$-th VAA is given by:

$$
f_{A M_{u}}=\sqrt{\frac{P_{R_{u}}}{P_{S} \cdot \sigma_{S R_{u}}^{2}+N_{0}^{R_{u}}}},
$$

Then, the signal received from the $u$-th VAA at the destination node, may be expressed as:

$$
\begin{aligned}
\mathbf{Y}_{n}^{R D_{u}} & =\sum_{m=1}^{M}\left(f_{A M_{u}} \mathbf{Y}_{n}^{S R_{u}^{m}} \mathbf{H}_{n}^{R D_{u}^{m}}\right)+\mathbf{V}_{n}^{R D_{u}} \\
& =f_{A M_{u}} \mathbf{Y}_{n}^{S R_{u}} \mathbf{H}_{n}^{R D_{u}}+\mathbf{V}_{n}^{R D_{u}} \\
& =\sqrt{P_{S}} f_{A M_{u}} \mathbf{S}_{n} \mathbf{H}_{n}^{S R_{u}} \mathbf{H}_{n}^{R D_{u}}+f_{A M_{u}} \mathbf{V}_{n}^{S R_{u}} \mathbf{H}_{n}^{R D_{u}}+\mathbf{V}_{n}^{R D_{u}}
\end{aligned}
$$

where $\mathbf{Y}_{n}^{S R} R_{u}$ is given by Eq. (3), and the AWGN matrix $\mathbf{V}_{n}^{R D_{u}}$ has a size of $(T \times N)$ elements with a zero mean and a variance of $N_{0}^{D}$. The $(M \times N)$-element matrix $\mathbf{H}_{n}^{R D_{u}}=\left[\left(\mathbf{H}_{n}^{R D_{u}^{1}}\right)^{T}, \cdots,\left(\mathbf{H}_{n}^{R D_{u}^{M}}\right)^{T}\right]^{T}$ in Eq. (6) models the fading channel of the $u$-th Relay-Destination (RD) link, which has a power of $\sigma_{R D_{u}}^{2}$.

Upon combining Eq. (4) and Eq. (6), a universal model for the received signal at the destination during both the broadcast interval and during the cooperation interval may be formulated as:

$$
\begin{array}{r}
{\left[\begin{array}{c}
\mathbf{Y}_{n}^{S D} \\
\mathbf{Y}_{n}^{R D_{2}} \\
\vdots \\
\mathbf{Y}_{n}^{R D_{U}}
\end{array}\right]=} \\
\quad\left[\begin{array}{cccc}
\mathbf{S}_{n} & \mathbf{0} & \cdot & \mathbf{0} \\
\mathbf{0} & \mathbf{S}_{n} & \cdot & \mathbf{0} \\
\vdots & \vdots & \ddots & \vdots \\
\mathbf{0} & \mathbf{0} & \cdots & \mathbf{S}_{n}
\end{array}\right]\left[\begin{array}{c}
\sqrt{P_{S}} \mathbf{H}_{n}^{S D} \\
\sqrt{P_{S}} f_{A M_{2}} \mathbf{H}_{n}^{S R_{2}} \mathbf{H}_{n}^{R D_{2}} \\
\vdots \\
\sqrt{P_{S}} f_{A M_{U}} \mathbf{H}_{n}^{S R_{U}} \mathbf{H}_{n}^{R D_{U}}
\end{array}\right] \\
+\left[\begin{array}{c}
\mathbf{V}_{n}^{S D} \\
f_{A M_{2}} \mathbf{V}_{n}^{S R_{2}} \mathbf{H}_{n}^{R D_{2}}+\mathbf{V}_{n}^{R D_{2}} \\
\vdots \\
f_{A M_{U}} \mathbf{V}_{n}^{S R_{U}} \mathbf{H}_{n}^{R D_{U}}+\mathbf{V}_{n}^{R D_{U}}
\end{array}\right],
\end{array}
$$

which may be modelled as:

$$
\mathbf{Y}_{n}=\hat{\mathbf{S}}_{n} \mathbf{H}_{n}+\mathbf{V}_{n} .
$$

If the VAA links fade slowly, then we may assume that the channel envelope and phase are nearly time-invariant over two consecutive blocks durations, i.e. we have $\mathbf{H}_{n+1} \approx \mathbf{H}_{n}$. Then the next received signal block may be expressed as:

$$
\begin{aligned}
\mathbf{Y}_{n+1} & =\hat{\mathbf{S}}_{n+1} \mathbf{H}_{n}+\mathbf{V}_{n+1} \\
& =\hat{\mathbf{X}}_{n} \hat{\mathbf{S}}_{n} \mathbf{H}_{n}+\mathbf{V}_{n+1} \\
& =\hat{\mathbf{X}}_{n}\left(\mathbf{Y}_{n}-\mathbf{V}_{n}\right)+\mathbf{V}_{n+1} \\
& =\hat{\mathbf{X}}_{n} \mathbf{Y}_{n}+\widetilde{\mathbf{V}}_{n+1},
\end{aligned}
$$

where the noise term $\widetilde{\mathbf{V}}_{n+1}=-\hat{\mathbf{X}}_{n} \mathbf{V}_{n}+\mathbf{V}_{n+1}$ may be minimized by the CDD as:

$$
\hat{\mathbf{X}}_{n}=\arg \min _{\hat{\mathbf{X}}_{n}}\left\|\mathbf{Y}_{n+1}-\hat{\mathbf{X}}_{n} \mathbf{Y}_{n}\right\|^{2}
$$



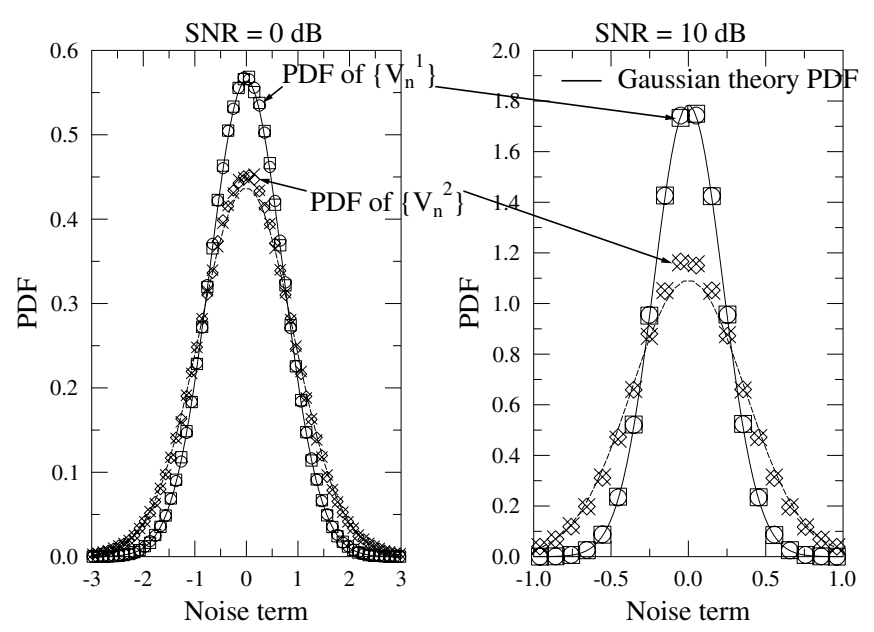

Fig. 2. The distribution of the noise term $\mathbf{V}_{n}$ in Eq. (8) at $S N R=0 \mathrm{~dB}$ and $S N R=10 \mathrm{~dB}$.

where $\hat{\mathbf{X}}_{n}=\operatorname{diag}\left\{\mathbf{X}_{n}, \cdots, \mathbf{X}_{n}\right\}$ has $(U T \times U T)$ elements. Eq. (10) shows that the CDD observes two received signal blocks and then makes a decision concerning a single $\hat{\mathbf{X}}_{n}$, again, under the assumption of a slowly faded channel.

\section{B. The MSDD/MSDSD}

In order to mitigate the potential performance degradation of CDD in fast fading channels, our MSDD/MSDSD observes $N_{w}$ received signal blocks and makes a joint decision based on the $\left(N_{w}-1\right)$ information matrices $\left\{\mathbf{X}_{i}\right\}_{i=1}^{N_{w}-1}$. Therefore, the received signal model of Eq. (8) may be written as:

$$
\mathbf{Y}=\mathbf{S H}+\mathbf{V}
$$

where the $N_{w}$-block based received signal matrix $\mathbf{Y}=$ $\left[\mathbf{Y}_{n-N_{w}+1}^{T}, \cdots, \mathbf{Y}_{n}^{T}\right]^{T}=\left[\mathbf{Y}_{1}^{T}, \cdots, \mathbf{Y}_{N_{w}}^{T}\right]^{T}$ has $\left(U T N_{w} \times\right.$ $N)$ elements. The accumulated fading channel matrix $\mathbf{H}=$ $\left[\mathbf{H}_{n-N_{w}+1}^{T}, \cdots, \mathbf{H}_{n}^{T}\right]^{T}$ and the accumulated noise matrix $\mathbf{V}=$ $\left[\mathbf{V}_{n-N_{w}+1}^{T}, \cdots, \mathbf{V}_{n}^{T}\right]^{T}$ in Eq. (11) have the sizes of $\left(U M N_{w} \times N\right)$ and $\left(U T N_{w} \times N\right)$, respectively. The $N_{w}$-block transmit signal matrix $\mathbf{S}=\operatorname{diag}\left\{\hat{\mathbf{S}}_{n-N_{w}+1}, \cdots, \hat{\mathbf{S}}_{n}\right\}=\operatorname{diag}\left\{\hat{\mathbf{S}}_{1}, \cdots, \hat{\mathbf{S}}_{N_{w}}\right\}$ has $\left(U T N_{w} \times U M N_{w}\right)$ elements.

The MSDD/MSDSD aims for maximizing the a posteriori probability of [11], [12]:

$$
\operatorname{Pr}(\mathbf{Y} \mid \mathbf{S}) \propto \exp \left(-\operatorname{Tr}\left\{\mathbf{Y}^{H} \mathbf{R}_{\mathbf{Y} \mathbf{Y}}^{-\mathbf{1}} \mathbf{Y}\right\}\right)
$$

which is based on the assumption that the noise term $\mathbf{V}$ of Eq. (11) obeys the Gaussian distribution. In the received signal model of Eq. (7), the noise term $V_{n}^{1}=V_{n}^{S D}$ of the SD links is an AWGN process having a zero mean and a variance of $N N_{0}^{D} \mathbf{I}_{\mathbf{T}}$, while the equivalent noise term $V_{n}^{2}=f_{A M_{2}} \mathbf{V}_{n}^{S R_{2}} \mathbf{H}_{n}^{R D_{2}}+\mathbf{V}_{n}^{R D_{2}}$ of the RD links may be considered as being near-Gaussian distributed with a zero mean and a variance of $N\left(N_{0}^{D}+f_{A M_{2}}^{2} \sigma_{R D_{2}}^{2} M N_{0}^{R_{2}}\right) \mathbf{I}_{\mathbf{T}}$ [15]. The probability density functions (PDFs) of the noise terms $\mathbf{V}_{n}^{1}$ and $\mathbf{V}_{n}^{2}$ are portrayed in Fig. 2, which verifies that the noise term $\mathbf{V}$ of Eq. (11) exhibits a Gaussian-like distribution.

The autocorrelation matrix $\mathbf{R}_{\mathbf{Y Y}}$ in Eq. (12) is given by:

$$
\begin{aligned}
\mathbf{R}_{\mathbf{Y Y}} & =\varepsilon\left\{\mathbf{Y} \mathbf{Y}^{H}\right\} \\
& =\mathbf{S R}_{\mathbf{H H}} \mathbf{S}^{H}+\mathbf{R}_{\mathbf{V V}},
\end{aligned}
$$

where the first transmit matrix $\hat{\mathbf{S}}_{1}$ in $\mathbf{S}$ is a common multiplier for all the following transmit matrices $\left\{\hat{\mathbf{S}}_{n}\right\}_{n=2}^{N_{w}}$, hence fixing $\hat{\mathbf{S}}_{1}$ or fixing the last one of the set, namely $\hat{\mathbf{S}}_{N_{w}}$ does not affect the search result of the MSDD/MSDSD. Therefore the accumulated differential matrices may be formulated as:

$$
\hat{\mathbf{A}}_{n}=\hat{\mathbf{S}}_{n} \hat{\mathbf{S}}_{N_{w}}^{H}= \begin{cases}\prod_{i=n}^{N_{w}-1} \hat{\mathbf{X}}_{i}^{H} & 1 \leqslant n<N_{w} \\ \mathbf{I}_{\mathbf{U T}} & n=N_{w} .\end{cases}
$$

Then Eq. (13) may be rewritten as:

$$
\begin{aligned}
\mathbf{R}_{\mathbf{Y Y}} & =\mathbf{A} \mathbf{R}_{\mathbf{H H}} \mathbf{A}^{H}+\mathbf{R}_{\mathbf{V V}} \\
& =\mathbf{A} \mathbf{C A} \mathbf{A}^{H},
\end{aligned}
$$

where $\mathbf{A}=\operatorname{diag}\left\{\hat{\mathbf{A}}_{1}, \cdots, \hat{\mathbf{A}}_{N_{w}}\right\}$ is a unitary matrix, and the channel's correlation matrix is defined as $\mathbf{C}=\mathbf{R}_{\mathbf{H H}}+\mathbf{R}_{\mathbf{V V}}$. According to Clarke's fading model, the correlation factors of the flat Rayleigh fading channels are determined by $\varphi(i)=\varepsilon\left\{h_{n} h_{n+i}^{*}\right\}=$ $J_{0}\left(2 \pi i f_{d}\right)$, where $J_{0}$ and $f_{d}$ denote the zero-order Bessel function of the first kind and the normalized Doppler frequency, respectively [12]. Therefore, the flat fading channel's autocorrelation matrix $\mathbf{R}_{\mathbf{H H}}$ in Eq. (15) is given by:

$$
\mathbf{R}_{\mathbf{H H}}=\left[\begin{array}{llll}
\boldsymbol{\Gamma}_{0} & \boldsymbol{\Gamma}_{1} & \ldots & \boldsymbol{\Gamma}_{N_{w}-1} \\
\boldsymbol{\Gamma}_{1} & \boldsymbol{\Gamma}_{0} & \ldots & \boldsymbol{\Gamma}_{N_{w}-2} \\
\vdots & \vdots & \ddots & \vdots \\
\boldsymbol{\Gamma}_{N_{w}-1} & \boldsymbol{\Gamma}_{N_{w}-2} & \ldots & \boldsymbol{\Gamma}_{0}
\end{array}\right]
$$

where each component is defined as $\boldsymbol{\Gamma}_{i}=$ $\operatorname{diag}\left\{\Gamma_{i}^{S D}, \boldsymbol{\Gamma}_{i}^{R D}\right\} \otimes \mathbf{I}_{\mathbf{T}}$ with $\Gamma_{i}^{S D}=N P_{S} \sigma_{S D}^{2} \varphi_{S D}(i)$ and $\Gamma_{i}^{R D}=\operatorname{diag}\left\{M N P_{S} f_{A M_{2}}^{2} \sigma_{S R_{2}}^{2} \sigma_{R D_{2}}^{2} \varphi_{S R_{2}}(i) \varphi_{R D_{2}}(i)\right.$, $\left.\cdots, M N P_{S} f_{A M_{U}}^{2} \sigma_{S R_{U}}^{2} \sigma_{R D_{U}}^{2} \varphi_{S R_{U}}(i) \varphi_{R D_{U}}(i)\right\}$. Furthermore, the noise correlation matrix $\mathbf{R}_{\mathbf{V V}}$ in Eq. (15) is formulated as:

$$
\begin{aligned}
\mathbf{R}_{\mathbf{V V}}= & \mathbf{I}_{\mathbf{N}_{\mathbf{w}}} \otimes \\
& {\left[\operatorname { d i a g } \left\{N N_{0}^{D},\left(N N_{0}^{D}+f_{A M_{2}}^{2} \sigma_{R D_{2}}^{2} M N N_{0}^{R_{2}}\right), \cdots,\right.\right.} \\
& \left.\left.\left(N N_{0}^{D}+f_{A M_{U}}^{2} \sigma_{R D_{U}}^{2} M N N_{0}^{R_{U}}\right)\right\} \otimes \mathbf{I}_{\mathbf{T}}\right] .
\end{aligned}
$$

According to Eq. (12), the MSDD is formulated as:

$$
\begin{aligned}
\mathbf{A} & =\arg \min _{\mathbf{A}} \operatorname{Tr}\left\{\mathbf{Y}^{H}\left(\mathbf{A} \mathbf{C} \mathbf{A}^{H}\right)^{-1} \mathbf{Y}\right\} \\
& =\arg \min _{\mathbf{A}} \operatorname{Tr}\left\{\mathbf{Y}^{H} \mathbf{A} \mathbf{C}^{-1} \mathbf{A}^{H} \mathbf{Y}\right\} \\
& =\arg \min _{\mathbf{A}}\left\|\mathbf{F} \mathbf{A}^{H} \mathbf{Y}\right\|^{2},
\end{aligned}
$$

where the upper triangular matrix $\mathbf{F}$ is generated from the decomposition of $\mathbf{C}^{-1}=\mathbf{F}^{H} \mathbf{F}$, which may be defined as:

$$
\mathbf{F}=\left[\begin{array}{llll}
\mathbf{F}_{1,1} & \mathbf{F}_{1,2} & \ldots & \mathbf{F}_{1, N_{w}} \\
\mathbf{0} & \mathbf{F}_{2,2} & \ldots & \mathbf{F}_{2, N_{w}} \\
\vdots & \vdots & \ddots & \vdots \\
\mathbf{0} & \mathbf{0} & \ldots & \mathbf{F}_{N_{w}, N_{w}}
\end{array}\right]
$$

Thus, the decision metric in Eq. (18) may be decomposed as:

$$
\left\|\mathbf{F A}^{H} \mathbf{Y}\right\|^{2}=\sum_{i=1}^{N_{w}}\left\|\sum_{j=i}^{N_{w}} \mathbf{F}_{i, j} \hat{\mathbf{A}}_{j}^{H} \mathbf{Y}_{j}\right\|^{2}
$$

which becomes a shortest vector problem for MSDSD [13]. More explicitly, the Partial Euclidean Distance (PED) from the received 


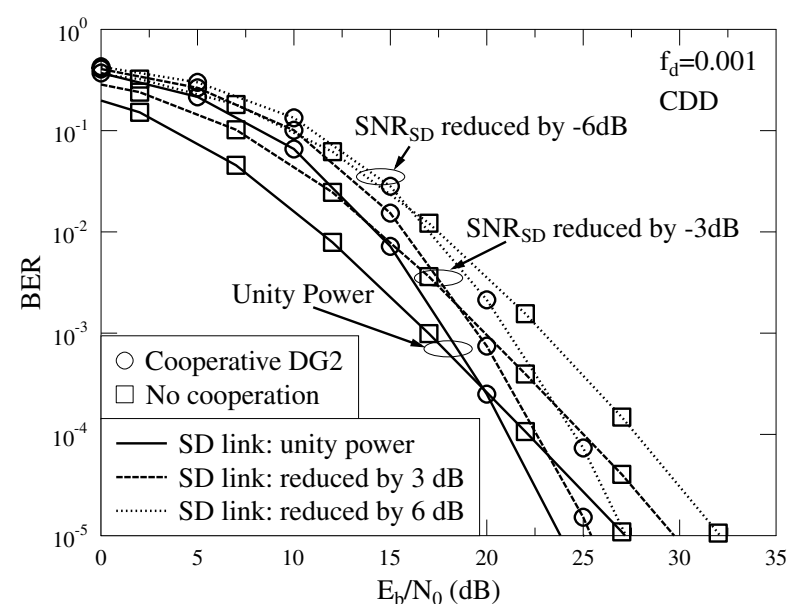

Fig. 3. BER performance of the CDD aided AF relaying DG2 employing QPSK when the power of SD link is faded, for $f_{d}=0.001$.

signal is expressed as:

$$
\begin{aligned}
d_{i}^{2} & =\sum_{t=i}^{N_{w}}\left\|\sum_{j=t}^{N_{w}} \mathbf{F}_{t, j} \hat{\mathbf{A}}_{j}^{H} \mathbf{Y}_{j}\right\|^{2} \\
& =d_{i+1}^{2}+\left\|\mathbf{F}_{i, i} \hat{\mathbf{A}}_{i}^{H} \mathbf{Y}_{i}+\sum_{j=i+1}^{N_{w}} \mathbf{F}_{i, j} \hat{\mathbf{A}}_{j}^{H} \mathbf{Y}_{j}\right\|^{2},
\end{aligned}
$$

with $i=1,2, \ldots,\left(N_{w}-1\right)$. Assuming that the DSTM scheme has $L$ legitimate codewords, each time when sphere decoding is performed, $L$ codewords corresponding to $\mathbf{X}_{i}$ are checked for minimizing $\boldsymbol{\Delta}_{i}=\mathbf{F}_{i, i} \hat{\mathbf{A}}_{i}^{H} \mathbf{Y}_{i}$ in Eq. (21). Note that the MSDD in Eq. (18) has to check all the $L^{N_{w}-1}$ possible combinations for $\mathbf{A}$, which results in an exponentially increasing complexity as $N_{w}$ increases. By contrast, the MSDSD characterized in Eq. (21) only has to check $L$ legitimate codewords for each individual $\left\{\mathbf{X}_{i}\right\}_{i=1}^{N_{w}-1}$, which sums to $L \times\left(N_{w}-1\right)$ codewords in total. It can be seen that MSDSD substantially reduces the decoding complexity of MSDD. The detailed sphere decoding algorithm may be found in [13].

\section{Simulation Results And Discussion}

In this section, we provide our simulation results for the proposed MSDSD aided AF relaying DSTM system. Without loss of generality, we consider the case of using a single VAA to assist the AF relaying system, i.e. we have $U=2$. Furthermore, we focus our attention on the DSTM scheme associated with $M=2$ transmit antennas at the source and $N=1$ receive antenna at the destination. We assume that the noise power imposed at the relay and at the destination are the same, since it was explored in [17] that the attainable performance of AF relaying schemes becomes better, when the relays are placed close to the destination.

Fig. 3 portrays the performance of the $\mathrm{CDD}$ aided AF relaying based Differential G2 (DG2) STBC employing QPSK modulation for transmission over slow fading channels associated with a normalized Doppler frequency of $f_{d}=f_{d}^{S D}=f_{d}^{S R}=f_{d}^{R D}=0.001$, where $f_{d}^{S D}, f_{d}^{S R}$ and $f_{d}^{R D}$ denote the normalized Doppler frequencies of $\mathrm{SD}, \mathrm{SR}$ and RD links, respectively. It can be seen that the cooperative scheme not only provides a higher diversity order, but also has a significant advantage over its co-located counterpart, when the power of the direct SD link is reduced. It is shown in Fig. 3 that each time the power of the SD link is reduced by $3 \mathrm{~dB}$, the overall performance

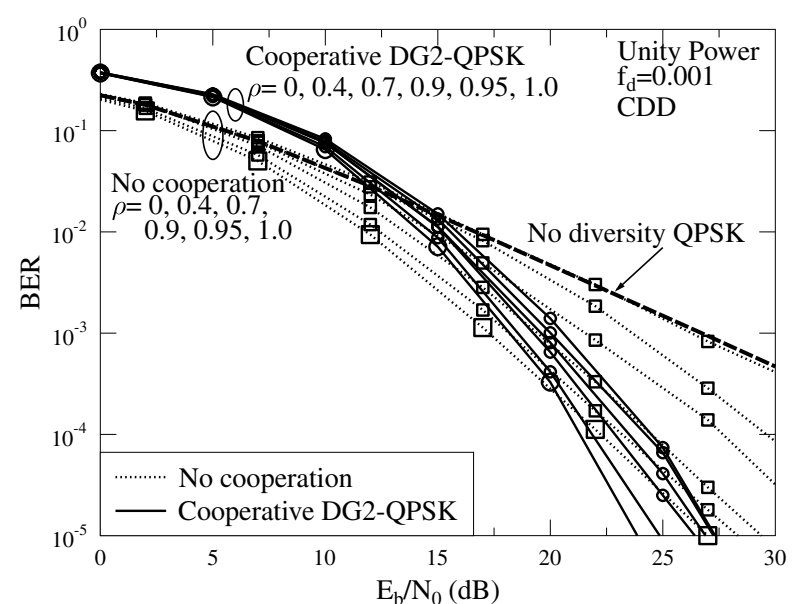

Fig. 4. BER performance of the CDD aided AF relaying DG2 employing QPSK experiencing antenna correlation at the source node, for $f_{d}=0.001$.

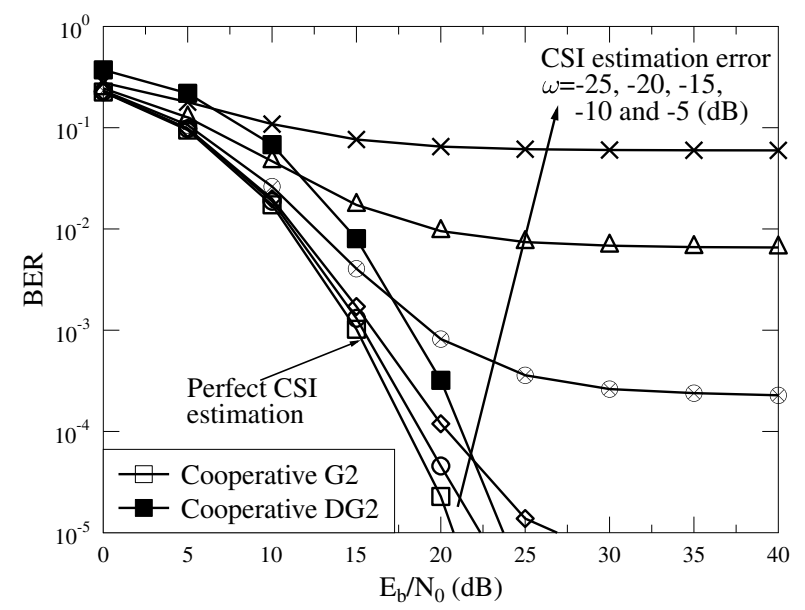

Fig. 5. BER performance of the CDD aided AF relaying DG2 STBC employing QPSK in comparison to its coherent counterpart associated with CSI estimation error, for $f_{d}=0.001$.

of the proposed AF scheme degrades only by about $1.5 \mathrm{~dB}$, since the SD link only contributes half of the overall diversity order.

Furthermore, when the SD link is in a deep shadow fade, spatial correlation is introduced between the co-located antenna elements at the source node. Fig. 4 characterizes the effect of spatial antenna correlation, where the spatial correlation model of [18] was adopted. Observe in Fig. 4 that the DG2 STBC employing QPSK modulation gradually loses its diversity gain over the single-input DQPSK scheme, as the spatial correlation $\rho$ between the two transmit antennas increases. By contrast, as a benefit of activating two extra relays experiencing independent fading, a diversity order of three is attained by the proposed AF relaying scheme, when the spatial correlation of the two transmit antennas at the source becomes $\rho=1$.

Fig. 5 compares our cooperative DG2 STBC employing QPSK modulation and its coherent counterpart. As the number of AA links increases, the estimation of CSI becomes more difficult, especially for the mobile-to-mobile channels. Fig. 5 demonstrates that when the CSI estimation is contaminated by the additive Gaussian noise of $C N(0, \omega)$, the $3 \mathrm{~dB}$ performance advantage of the coherent scheme was eroded. By contrast, our cooperative DSTM scheme does not require channel estimation for the distributed links. 


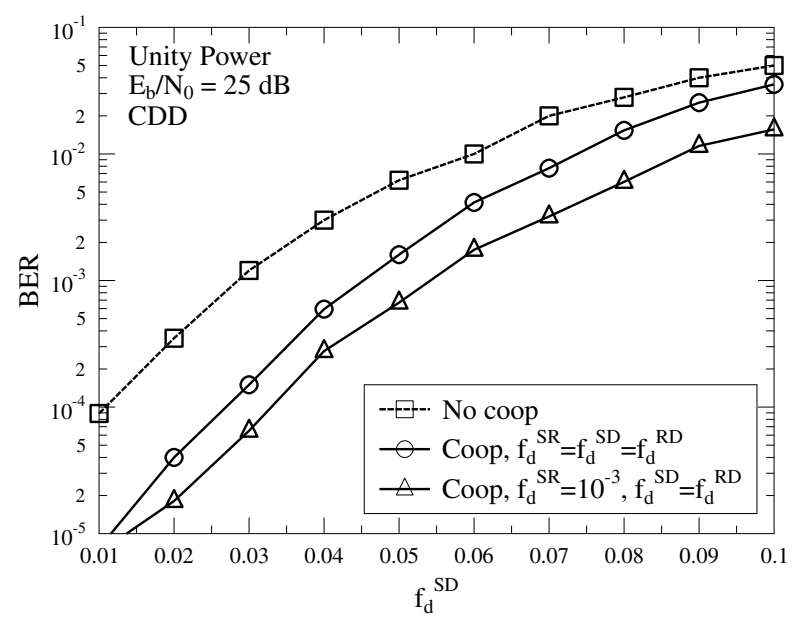

Fig. 6. Error floor of the CDD aided AF relaying DG2 employing QPSK at $E_{b} / N_{0}=25 \mathrm{~dB}$ and different normalized Doppler frequencies.

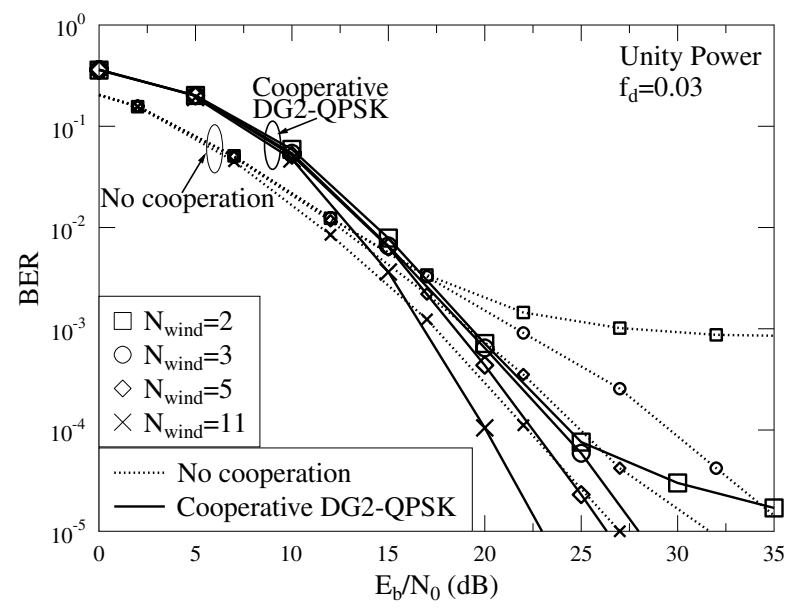

Fig. 7. BER performance of the MSDSD aided AF relaying Differential G2 (DG2) employing QPSK, for $f_{d}=0.03$.

However, the CDD of our cooperative DSTM proposed in Section II-A operates under the assumption that the fading channel fluctuates slowly. Upon increasing the Doppler frequency, an irreducible error floor may form, which is portrayed by Fig. 6. It can be seen that the CDD aided AF relaying associated DG2 STBC has a lower error floor in rapidly fading channels than the co-located DG2 STBC operating without relays. Furthermore, if the SR link is in slow fading, i.e. we have $f_{d}^{S R}=0.001$, then the error floor is further reduced. Hence it is beneficial for the BS to aim for activating relays, which are not moving rapidly. In Fig. 7, we apply the MSDSD proposed in Section II-B at a normalized Doppler frequency of $f_{d}=f_{d}^{S D}=f_{d}^{S R}=f_{d}^{R D}=0.03$. It can be seen that the irreducible error floor of the CDD recorded in rapidly fading channels is successfully mitigated by our proposed MSDSD. Furthermore, the MSDSD aided AF relaying associated DG2 scheme outperforms its non-cooperative counterpart by $3 \mathrm{~dB}$ in conjunction with an MSDSD window length of $N_{w}=11$ at the BER level of $10^{-5}$, since the cooperative diversity order is doubled with the aided of a single VAA in comparison to the co-located DSTM.

\section{CONCLUSIONS}

In this paper, we proposed an AF relaying aided DSTM scheme, which outperforms its co-located counterpart, especially when the source node is subjected to deep shadow fades and hence the signals of the different transmit antennas become spatially correlated. Our simulation results demonstrated that the coherent counterpart of our cooperative DSTM scheme suffers from CSI estimation errors, which erodes its $3 \mathrm{~dB}$ performance advantage. Furthermore, we developed a MSDSD for the proposed cooperative scheme in order to mitigate the typical irreducible error floor of noncoherent receivers operating in fast fading channels.

\section{REFERENCES}

[1] D. Gesbert, M. Shafi, D. S. Shiu, P. Smith, and A. Naguib, "From theory to practice: An overview of MIMO space-time coded wireless systems," IEEE Journal on Selected Areas in Communications, vol. 21, pp. 281 302, April 2003.

[2] A. Goldsmith, S. Jafar, N. Jindal, and S. Vishwanath, "Capacity limits of MIMO channels," IEEE Journal on Selected Areas in Communications, vol. 21, pp. 684 - 702, June 2003.

[3] S. Alamouti, "A simple transmit diversity technique for wireless communications," IEEE Journal on Selected Areas in Communications, vol. 16, pp. $1451-1458$, Oct. 1998 .

[4] V. Tarokh, H. Jafarkhani, and A. Calderbank, "Space-time block codes from orthogonal designs," IEEE Transactions on Information Theory, vol. 45, pp. 1456 -1467, July 1999.

[5] J. Heath, R.W. and A. Paulraj, "Linear dispersion codes for MIMO systems based on frame theory," IEEE Transactions on Signal Processing, vol. 50 , pp. 2429 - 2441, Oct. 2002

[6] J. Laneman and G. Wornell, "Distributed space-time-coded protocols for exploiting cooperative diversity in wireless networks," IEEE Transactions on Information Theory, vol. 49, pp. 2415-2425, Oct. 2003.

[7] A. Sendonaris, E. Erkip, and B. Aazhang, "User cooperation diversity. Part I. System description," IEEE Transactions on Communications, vol. 51, pp. 1927-1938, Nov. 2003.

[8] V. Tarokh and H. Jafarkhani, "A differential detection scheme for transmit diversity," IEEE Journal on Selected Areas in Communications, vol. 18, pp. $1169-1174$, July 2000.

[9] H. Jafarkhani and V. Tarokh, "Multiple transmit antenna differential detection from generalized orthogonal designs," IEEE Transactions on Information Theory, vol. 47, pp. 2626 -2631, Sept. 2001.

[10] B. Hassibi and B. Hochwald, "Cayley differential unitary space-time codes," IEEE Transactions on Information Theory, vol. 48, pp. 1485 -1503 , June 2002.

[11] D. Divsalar and M. Simon, "Multiple-symbol differential detection of MPSK," IEEE Transactions on Communications, vol. 38, pp. 300-308, Mar. 1990.

[12] P. Ho and D. Fung, "Error performance of multiple-symbol differential detection of PSK signals transmitted over correlated Rayleigh fading channels," IEEE Transactions on Communications, vol. 40, pp. 15661569, Oct. 1992.

[13] L. Lampe, R. Schober, V. Pauli, and C. Windpassinger, "Multiple-symbol differential sphere decoding," IEEE Transactions on Communications, vol. 53, pp. 1981-1985, Dec. 2005.

[14] V. Pauli and L. Lampe, "Tree-search multiple-symbol differential decoding for unitary space-time modulation," IEEE Transactions on Communications, vol. 55, pp. 1567-1576, Aug. 2007.

[15] L. Wang and L. Hanzo, "The amplify-and-forward cooperative uplink using multiple-symbol differential sphere-detection," IEEE Signal Processing Letters, vol. 16, pp. 913 -916, October 2009.

[16] L. Hanzo, O. Alamri, M. El-Hajjar, and N. Wu, Near-Capacity MultiFunctional MIMO Systems - Sphere-Packing, Iterative Detection and Cooperation. Wiley-IEEE Press, 2009.

[17] L. Wang and L. Hanzo, "The resource-optimized differentially modulated hybrid AF/DF cooperative cellular uplink using multiple-symbol differential sphere detection," IEEE Signal Processing Letters, vol. 16, pp. $965-968$, Nov. 2009.

[18] R. Ertel and J. Reed, "Generation of two equal power correlated Rayleigh fading envelopes," IEEE Communications Letters, vol. 2, pp. 276 -278, Oct. 1998. 